\title{
Realidad y Ficción en Hijo de Hombre
}

Roa Bastos define el propósito de su novela, Hijo de Hombre (1959), de la siguiente manera: "Su tema trascendente al margen de la anécdota, es la crucifixión del hombre común en la lucha por su libertad, librado a sus solas fuerzas en un mundo y en una sociedad inhumanos que son su negación.' " Dada la evidente militancia subversivá, Roa Bastos evita todo posible folletinismo o propaganda a través de dos aspectos importantes: (1) enlaza las tramas individuales de sus personajes al periodo de historia paraguaya que va desde la dictadura del Dr. Francia (1814) hasta la guerra del Chaco (1932-35), creando así una perspectiva épica desde realidades irrefutables; (2) la novela consta de las memorias/confesiones del teniente Miguel Vera que se desarrolla como la antítesis del ideal humano heroico-solidario de Roa Bastos.

Este segundo aspecto es la base estructural y conflictiva sobre la que se centra la temática de Roa Bastos, y cuyo "sistema" " es el propósito de este estudio.

En el primer capitulo hay un claro intento de mantener la presencia del narradớ (el "yo") a un mínimo absoluto para dejar emerger sin distracciones a "la memoria viviente del pueblo", 2 -Macario Francia-- que es quien expone en simples palabrảs el tetna trascendente de la búsqueda de la solidaridad: "El hombre, mis hijos..., es como un ro. Tiene barranca y orilla. Nace y desemboca en otros ríos. Alguna utilidad debe prestar. Mal río es el que muere en un estero...' (p. 15) Y, más adelante, al explicar por qứ el cerro de Itapé con su Cristo leproso debiera llamarse Camino-del-Hombre (Kuimbaé-Rápé), Macario dice: “Porque el hombre, mis hijos..., tiene dos nacimientos. Uno al nacer, otro al morir...Muere pero queda vivo en los otros, si ha sido cabal con el prójimo. Y si sabe olvidarse en vida de sí mismo, la tierra come su cuerpo pero no su recuerdo..." (p. 38) Desde el principio, Macario, en esta función suya de “memoria viviente”' enlaza así el

Boletin de la Editorial Losada, Negro sobre Blanco, No. 10 (1959), p. 10. Pasaje citado por H. RodriguezAlcalá, "Hijo de bombre de Roa Bastos y la intrahistoria del Paraguay," Cuadernos Americanos, 127 (1963), p. 224.

${ }^{2}$ A. Roa Bastos, Hijo de hombre, 3a ed. (Buenos Aires: Losada, 1967), p. 14. Las siguientes citas de esta obra provienen de la misma edición y se indicará el número de la păgina junto a cada cita. 
marco histórico-recordatorio con el ideal humano individual y colectivo. Hay que observar que el núcleo psicológico de la novela en torno al cual se desarrolla la significación individual de los siete personajes principales (Miguel Vera, Macario Francia, Alexis Dubrovsky, Casiano Jara, Cristóbal Jara y Crisanto Villalba) es precisamente el "saber olvidarse en vida de sí mismo"; principio que en boca del centenario Macario asume el carácter de un mandato o ideal secular que se irá verificando a distintos niveles en cada personaje y bifurcándose en dos vertientes antitéticas. Macario también concluye que "tiene que ser obra de todos" (p. 38) proyectando así la responsabilidad individual a un plano colectivo: una de las condiciones épicas.

Los nueve episodios relativamente autónomos de los que consta Hijo de Hombre tienen como elemento unitivo al narrador, Miguel Vera, el cual alterna el uso de la primera persona (capitulos 1, 3, 5, 7, y 9) con el de la tercera persona (capítulos 2, 4, 6, y 8). ${ }^{3}$ Esta oscilación sistemática tiene una doble función estructural y temática que puede adaptarse a un esquema rigurosamente simétrico. En efecto, el mismo Vera define en dos instancias aisladas su carácter de narrador que proyecta su vida y experiencias a veces hacia un lector anónimo, mientras que, en otras ocasiones, él se desdobla para asumir la doble personalidad de narrador-lector: "Mi testimonio no sirve más que a medias. Ahora mismo, mientras escribo estos recuerdos, siento que a la inocencia, a los asombros de mi infancia, se mezclan mis traiciones y olvidos de hombre, las repetidas muertes de mi vida. No estoy reviviendo estos recuerdos; tal vez los estoy expiando.' (p. 14) Estas palabras parecen, entonces, determinar la actitud general de los capítulos especitícamente autobiográficos, a través de los cuales se observa detalle tras detalle la posición vacilante de Miguel Vera; su incapacidad de tomar una posición de verdadero compromiso en ningún momento de su vida es lo que le hace encarnar a la inversa el principio de Macario: Vera es el "mal río", el que no "sabe perderse en vida de sí mismo." Como dice RodriguezAlcalá: "...el relator Miguel Vera, personaje que, no siendo el héroe de un libro en que hay héroes auténticos como Casiano Jara y su hijo Cristóbal, representa en la sociedad de hoy al intelectual vacilante y cobarde, incapaz de solidarizarse por entero con los oprimidos, a quienes, sin embargo, comprende y compadece." ${ }^{4}$ Vera es entonces el elemento de contraste, el anti-héroe cuya constante introspección intelectualista lo amarra a su propio ser intrínseco y le lleva a cometer una serie de traiciones y autotraiciones: los capítulos impares a partir del tercero son esta sucesión episódica de su propia personalidad a través de momentos claves de su vida.

En los capitulos pares con la narración en tercera persona, es nuevamente Vera quien alude a su doble función de narrador-lector: "Veo el vapor que mana de mi cuerpo, mientras anoto estas cosas en mi libreta. ¿Por qué lo hago? Tal vez para releerlas más tarde, al azar. Tienen entonces un aire de divertida irrealidad, como si las hubiera escrito otro. Las releo en voz alta, como si alguien me contara cosas desconocidas para mí." (p.

\footnotetext{
${ }^{3}$ D. W. Foster, en su artículo "Nota sobre el punto de vista narrativo en Hijo de bombre de R. B., Revista Iberoamericana, 36 (1970), 643-650, presenta la hipótesis de que los capitulos pares son obra de otro narrador, cuya personalidad es ajena a la de Vera. Si bien no estoy de acuerdo con su opinión, creo que se le debe considerar en el estudio de Roa Bastos.

4 “'Hijo de hombre de R. B. y la intrahistoria del Paraguay," 127 (1963), 224.
} 
170) Si los capitulos impares marcan la experiencia vital de Vera, la lectura detenida de los capítulos pares también suponen un proceso sistemático: la creación del hombre que "sabe olvidarse en vida de sí mismo," el héroe total y solidario que es Cristóbal Jara. En efecto, el ruso Dubrovsky parece apuntar hacia lo ideal pero la avaricia lo aparta radicalmente del camino solidario (capítulo $2^{\circ}$ ). La huida épica de Casiano y Nati Jara de los yerbales --cuyo arranque inicial fue la salvación del hijo Cristóbal-- seconvierte en una obsesión que raya en la demencia al centrarse en empujar al vagón del tren a través de la selva; si bien el vagón se convierte en un mito colectivo de gran significación simbólica -sobre todo al enlazarse visualmente con el camión de Cristóbal en el desierto-- es evidente que el olvido de sí mismo de Casiano se diluye en una huida constante intrínsecamente antisocial (capitulo $4^{\circ}$ ). En el capitulo $6^{\circ}$, que proviene directamente de la traición de Vera, el carácter heroico de Cristóbal se va definiendo a través de su huida de las fuerzas gubernamentales y a través del recuerdo de los compañeros presos. Hay que notar que aquí también Vera sufre las consecuencias de su asociación con los revolucionarios y se esconde detrás de la narración en tercera persona para no asumir la responsabilidad directa de su traición. En el capítulo $8^{\circ}$, ya Cristóbal Jara asume plenamente la responsabilidad del "olvido en vida de sí mismo" con plena conciencia de su misión solidaria: Jara es entonces el héroe completo que se ha venido forjando dolorosamente mediante cuatro episodios sucesivos y una historia propia.

De este breve análisis resulta clara la imposibilidad de fundir la creación del héroe con la vida del antihéroe: el mismo intelectualismo narcisista de Vera es lo que le hace comprender su propia condición y es como si no quisiera contaminar con sus repetidas deserciones la gradual modelación del héroe como queda finalmente definido individual y colectivamente en Cristóbal Jara:

Eso era el destino. Y qué podía ser el destino para un hombre como Cristóbal Jara, sino conducir su obsesión como un esclavo por un angosto pique en la selva o por la llanura infinita, colmada con el salvaje olor de la libertad. Ir abriendo paso en la inexorable maraña de los hechos, dejando la carne en ella, pero transformándolos también con el elemento de esa voluntad cuya fuerza crecía precisamente al integrarse en ellos. 'Lo que no puede hacer el hombre, nadie más puede hacerlo'..., habia dicho él mismo. No estribaba acaso su fuerza en la simplicidad de acatar una ley que los incluía y los sobrepasaba. No sabían nada, ni siquiera tal vez lo que es la esperanza. Nada más que eso: querer algo hasta olvidar todo lo demás. Seguir adelante, olvidándose de sí mismos. Alegría, triunfo, derrota, sexo, amor, desesperación, no eran más que eso: tramos de la marcha por un desierto sin límites. Uno caía, otro seguía adelante, dejando un surco, cada huella, un rastro de sangre sobre la vieja costra, pero entonces la feroz y la elemental virgindad quedaba fecundada. (p. 247).

Frente a esta potencialidad y realidad heroico-histórica Vera no puede y no debe unir su historia a la de los demás (excepto en el capítulo quinto donde ni la heroicidad de Jara ni su propia anti-heroicidad han sido definitivamente delineadas); aún ya hacia el final de su vida, al darse cuenta de la necesidad absoluta de un cambio político y social radical, Vera se 
mantiene al margen:

Algo tiene que cambiar. No se puede seguir oprimiendo a un pueblo indefinidamente. El hombre es como un río, mis hijos..., decía el viejito Macario Francia. Nace y muere en otros ríos. Mal río es el que muere en un estero...El agua estancada es ponzoñoza. Engendra miasmas de una fiebre maligna, de una furiosal locura. Luego, para curar al enfermo o apaciguarlo, hay que matarlo. $\mathrm{Y}$ el suelo de este país ya está bastante ocupado bajo tierra: “"Los muertos bajo tierra no prenden!..."

Temo que un dia de estos vengan a proponerme, como allá en Sapukai, que les enseñe a combatir. Yo a ellos..., ;qué escarnio! Pero no, ya no lo necesitan. Han aprendido mucho. El camión de Cristóbal Jara no atravesó la muerte para salvar la vida de un traidor. Envuelto en las llamas sigue rodando en la noche, sobre el desierto, en las picadas, llevando el agua para la sed de los sobrevivientes. (p. 274).

Es evidente que la amarga exclamación, “iqué escarnio!'” de Vera frente a la remota posibilidad de otra asociación suya con los revolucionarios hace patente su propia función de contrapunto anti-heroico. Además, al exteriorizar totalmente las historias narradas en los capítulos pares, al utilizar la tercera persona narrativa, Vera se enajena, se independiza aún más del ejemplo humanamente válido que cada uno de los otros personajes le ofrece; de modo que, al ficcionalizarlos de su propia realidad, él logra --una vez más-- evadir la propia responsabilidad.

Este proceso de exteriorización en el cual Vera vuelve a mitificar para sí mismo personajes y situaciones (Dubrovsky, explosión de Sapukai, Casiano y Nati Jara) que pertenecen ya a la mitología y al folklore local, es llevado a sus últimas consecuencias en cuanto que Vera, en su afán de evasión, llega a ficcionalizar sus propias experiencias. En efecto, en la segunda parte del capitulo 6 (Fiesta), Vera anula la responsabilidad directa de su traición al incluir su escena del calabozo en el episodio narrado en tercera persona:

--iTeniente Vera!... --barbotó el oficial-- ¿Me ha oido? --lo removió con la punta de la bota.

--Yo no sé nada... -- dijo solamente sin volver la desgreñada cabeza...

--Lo que se dice en una borrachera noitiene valor... -- la voz opaca se asordinó aún más contra la pared de ladrillos. ...

--Yo no delaté a esos hombres... -- dijo otra vez la voz monótona y lejana...

No repitió, ni intentó aclarar lo que dijo, si era que acababa de decir alguna cosa...

--No sé nada....iDéjenme en paz! -- tornó a repetirla voz incolora con un resabio de amargura y de asco.

(pp. 139-141)

La posibilidad de que el diálogo o el capítulo entero hayan sido escritos por un segundo narrador queda eliminada en cuanto que el fragmento mismo incluye la clave del desdoblamiento de Vera, de su carácter de hombre "partido": "La puerta entornada del

${ }^{5}$ El subrayado es mío. 
calabozo le dejaba caer en mitad del pecho una polvorienta barra de sol que partía su cuerpo en dos pedazos sombrios," (p. 138) y, más adelante se repite la misma imagen esclarecedora: "Se escuchó otra vez el gorgoteante murmullo, pero los dos pedazos del hombre siguieron inmóviles sin más que ese lento balanceo del pecho bajo la barra del sol..." (p. 140) Es más, la ficcionalización de la culpa funciona como una extensión narrativa de la traición llevada a cabo en el enajenamiento de la borrachera.

Hasta el capítulo 5, a pesar de varias indicaciones, el carácter de Vera todavía podía haberse manifestado hacia una actidud solidaria, sobre todo si se considera su mentalidad lúcida; pero, a partir de este "delito" queda irrevocablemente definido en una sentimentalidad pasiva, rayante en el cinismo.

Es este, entonces, el eje narrativo que marca definitivamente la bifurcación de las dos vertientes antitéticas: el héroe (Gaspar Mora, Dubrovsky, Casiano Jara y Cristóbal Jara, progresivamente) y el anti-héroe (Vera). Es de notar que sólo ahora se da a conocer el nombre de Miguel Vera: último detalle necesario para completar su individualización.

Queda por resolver la significación del primer capitulo donde a través de la "memoria viviente"' de Macario se pasa una revista subjetiva y mítica a toda la historia del Paraguay independiente hasta 1912 (fecha de la consagración del Cristo leproso de Itapé); además es en este mismo episodio inicial donde Macario hace revivir la misión de Gaspar Mora. Se observa entonces que la historia relatada por Macario es ese corpus de tradiciones y de herencias comunes, la raíz de donde arranca el presente de la novela, el seno de donde parten cada uno de los personajes para formar un futuro ya sea solidario o anti-solidario. Gaspar, por el otro lado, define la filosofía, la teoría de la solidaridad en todo su sentido "fieramente humano": "Gaspar representa a un Cristo pasivo, sufrido y religioso cuyo espíritu sobrevive en una estatua de madera; en cambio, Cristóbal es el redentor ateo con raíces en la cultura guaraní y cuya determinación inquebrantable frente a los obstáculos más espantosos se inmortalizará en todas las sublevaciones que sean necesarias para conseguir la libertad y la justicia." " Si Vera-por decirlo así-se "atreve" a relatar este primer episodio en primera persona es porque aún era niño inocente y "creía firmemente en algo" (p. 23): en la validez y en la pureza del mensaje de Gaspar Mora; no es hasta el tercer capítulo donde se empieza a manifestar su carácter vacilante: "Yo era un desertor. Sentía tristeza y vergüenza, a pesar de las ropas, de los zapatos, del viaje, de la escuela lejana, del futuro honor de cadete, más lejano todavia." (p. 63). Hay que notar, sin embargo, que aqui Vera diluye su participación individual en las acciones colectivas de la chiquillería del pueblo, anulándose, de modo que lo que verdaderamente predomina es la primera persona del plural: "Algunos lo seguíamos no para tirarle tierra sino para oir sus relatos y sucedidos, que tenían el color y el sabor de lo vivido;" (p. 14) "lo escuchábamos con escalofríos;" (p.15) "no le entendíamos muy bien;"' (p. 15) "otro día nos llevó a su rancho;"' (p. 18) "En ese momento comprendíamos también las palabras rotas de María Rosa. En su dulce obsesión adivinábamos la parte en sombras de la historia de Gaspar"' (p. 21) y otros varios casos más.

${ }^{6}$ Seymour Menton, "Realismo mágico y dualidad en Hijo de bombre," Revista Iberoamericana, 33 (1967), 
La bifurcación general de la obra se puede esquematizar gráficamente de la siguiente manera:

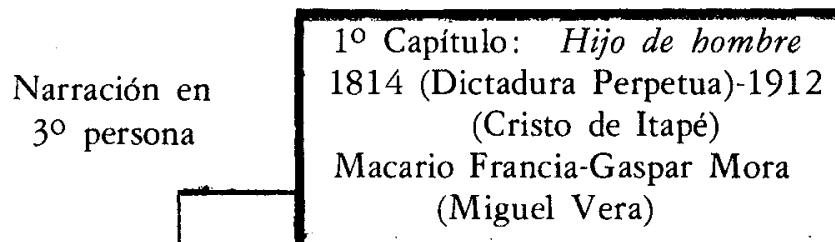

$2^{\circ}$ Cap.: Madera y carne

Alexis Dubrovsky (1917-22?)

María Regalada Caceré

Descripción de la estación de Sapukai ( $1^{\circ}$ de marzo 1912) Mención del vagón de Casiano y Nati Jara

$4^{\circ}$ Cap.: Exodo (1912-1914)

Fuga de Casiano y Nati Jara

de los yerbales

Descripción de la explosión de Sapukai

Mención del viaje del vagón de Casiano y Nati Jara

$6^{\circ}$ Cap.: Fiesta (1931)

Fuga de Cristóbal Jara

Encarcelamiento de Vera

María Regalada (su hijo Alejo)

Baile de los lazarientos

$8^{\circ}$ Cap.: Misión (1932)

Cristóbal asume su misión

Silvestre Aquino-Salu'i

Camión de Cristóbal se

funde con el vagón de

Casiano

Muerte de Cristóbal-mito $3^{\circ}$ Cap.: Estaciones

Miguel Vera (1917)

Descripción de la explosión

de Sapukai (1912)

Mención del vagón de Casiano

y Nati Jara

Primera traición de Vera

$5^{\circ}$ Cap.: Hogar (1931)

Miguel Vera-Cristóbal Jara

Descripción de la explosión

de Sapukai

Descripción del viaje del vagón de C. y N. Jara

Segunda traición de Vera

Sugerencia de la tercera

traición de Vera

$7^{\circ}$ Cap.: Destinados (1932)

Vera en Peña Hermosa (penal)

Cuarta traición de Vera

Guerra del Chaco-Boquerón

Vera mata a Jara

$9^{\circ}$ Cap.: Ex-combatientes (1936)

Vera-alcalde de Itapé

Crisanto Villalba (Jocó)

Muerte (suicidio) de Vera

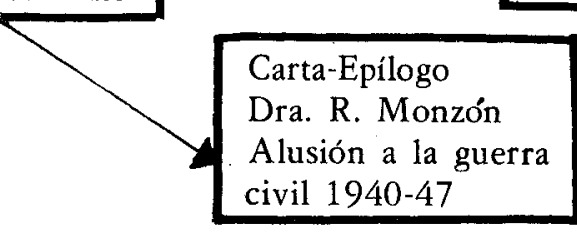


El nivel temporal que enmarca las memorias/confesiones de Vera se encuentra definido por un "hoy" y un " ahora" que se pueden fechar en un periodo inmediatamente posterior a la Guerra del Chaco (1932-35), dada la descripción del crecimiento material y del ambiente de progreso provinciano del pueblo de Itapé: "Ahora los trenes pasan más a menudo. Hay una estación nueva... Un ramal conduce a la fábrica de azúcar que se ha levantado sobre el río... La iglesia nueva recubre los muñones de la antigua..." (p. 12) También, como se sabrá en el último capítulo (Ex-combatientes), Vera es alcalde de Itapé reemplazando a Melitón Isasi, que había ejercido el puesto durante la guerra (p. 260), cuando Crisanto Villalba regresa un año después del Desfile de la Victoria (p. 255).

El tono expiatorio y de autoenjuiciamiento que predomina en los capítulos $1,3,5$ y $9^{7}$ enfatiza el hecho que Vera está pasando revista a su vida después de la experiencia total de todas sus traiciones antes de responsabilizar a Cuchuí con su muerte/suicidio. Debe de haber escrito también el capítulo 8 (Misión) en el periodo de post-guerra ya que pudo haber conocido la evolución heroico-mítica de Cristóbal Jara sólo después de su tratamiento terapéutico con la Dra. Monzón.

El otro nivel temporal del narrador lo marca claramente el diario de Vera, que incluye sus experiencias en el penal de Peña Hermosa y en la Guerra del Chaco. Si bien el diario abarca un periodo desde el primero de enero de 1932 al 29 de septiembre del mismo año, hay claras indicaciones que su estadia en el penal comenzó hace ya algún tiempo.

En la primera entrada del diario dice: "Veo el vapor que mana de mi cuerpo, mientras anoto estas cosas en mi libreta. ¿ Por qué lo hago? Tal vez para releerlas más tarde, al azar. Tienen entonces un aire de divertida irrealidad, como si las hubiera escrito otro. Las releo en voz alta, como si conversara con alguien, como si alguien me contara cosas desconocidas por mí. Sin embargo, hasta escribir me cansa.' (p. 170) Dada la obsesión autobiográfica de Vera, resulta difícil pensar que se está refiriendo a "cosas" como el diario mismo y que no las haya conservado; además, el "aire de divertida irrealidad" es totalmente incongruente con la solemnidad simbológica con que trata su propia vida y pensamientos. Se observa que Vera da aquí una clave en cuanto a la transformación de su perspectiva: de una histórico-personal a otra literario-personal. Por otro lado, es enteramente posible especular que se hubiera dedicado a recrear mitos locales surgidos en un pasado próximo. El mismo dice en el capítulo 5, al hacer el viaje "turístico" con Cristóbal Jara al vagón enterrado en la selva, que estaba enterado de las historias-leyendas de Dubrovsky (pp. 117-118) y de Casiano y Nati Jara (pp. 122-125). También pudo haber escrito el capítulo 6 (Fiesta), el de

Cap. 1: "Mi testimonio no sirve más que a medias. Ahora mismo, mientras escribo estos recuerdos, siento que a la inocencia, a los asombros de mi infancia, se mezclan mis traiciones y olvidos de hombre, las repetidas muertes de mi vida. No estoy reviviendo estos recuerdos; tal vez los estoy expiando." (p. 14) "...contaba cambiándola un poco cada vez. Superponía los hechos, trocaba nombres, fechas, lugares, como quizás lo esté haciendo yo ahora sin darme cuenta, pues mi incertidumbre es mayor que la de aquel viejo chocho, que por lo menos era puro." (p. 20). Cap. 3: "Yo era un desertor. Sentia tristeza y verguienza..." (p. 63) Cap. 5: "...robando la leche del crío enfermo que dormía apretado en sus brazos, traicionando también a medias al marido emparedado en la cárcel. Así yo había descubierto el triste amor en la oscuridad junto a unas ruinas, como un profanador o un ladrón en la noche." (pp. 127-128)

$\checkmark$ “....apenas nos apercibimos del paso del tiempo... La lancha del Resguado hace su arribo mensual con los viveres y la correspondencia... El mes pasado llegó el último..." (p. 168) "Se repiten los pequeños actos anónimos de hostilidad." (p. 171) 
su propia ficcionalización ("como si las hubiera escrito otro"), ya que la fuga de Cristóbal se efectúa en el giro de un día y una noche y Vera, seguramente, supo los detalles antes de ser enviado al penal.

En la carta-epílogo, la Dra. Monzón dice " he copiado los papeles de Vera sin cambiar nada, sin alterar una coma. Sólo he omitido los párrafos que me conciernen personalmente; ellos no interesan a nadie." (p. 281) De modo que la organización final de los capítulos con su alternancia simétrica se debe a Vera: de acuerdo con el esquema gráfico presentado, es evidente que los capítulos impares a partir del tercero (y también del primero, en lo que atañe directamente a Vera) siguen el progreso rigurosamente cronológico de su vida, mientras que los pares $(2,4,6$ y 8$)$ van creando-independientemente de la cronología-el héroe total, activo y solidario que se mitifica en la historia y en la intra-historia del Paraguay.

La obra es entonces una dicotomía estructural ("buen río"/"mal rio", héroe/antihéroe, solidaridad/antisolidaridad) que se desarrolla dialécticamente, testimoniando y presagiando "a este pueblo tan calumniado de América, que durante siglos ha oscilado sin descanso entre la rebeldía y la opresión, entre el oprobio de sus escarnecedores y la profecía de sus mártires..." (p. 281) 